\title{
Correlation of Polarized Light Phenomena With the Orientation of Some Metal Crystals
}

\author{
C. J. Newton and H. C. Vacher
}

\begin{abstract}
A photometric study was made of the reflection of plane polarized light normally incident in a metallographic microscope upon specimens of tin, aluminum, and monel, with various surface treatments. A high degree of correlation was found in most cases between the extinction position and the projection of the optic axis or of the cubic axis making the greatest angle with the surface normal. Moreover, a fourth power sine relationship was observed between the change of intensity and the angle between the surface normal and the optic axis in tin. The intensity correlation in the case of the cubic metals was not so clear, but a slight indication of position dependence was observed for aluminum. The results indicate that the optically anisotropic effects observed with cubic metals can be caused both by anisotropic films and by oriented surface contours, but that the latter is the source of those effects that are correlated with the crystallographic orientation of the grain.
\end{abstract}

\section{Introduction}

In continuation of an investigation $[1,2]^{2}$ of the utility of plane polarized light in metallography, a study has been made of tin and aluminum crystals, and additional data have been obtained from monel, a 70 nickel-30 copper alloy. The previous work at the National Bureau of Standards by D. H. Woodard [1] showed a nonuniformity of intensity of polarized light reflected from plastically deformed metal grains, suggesting an observable correlation between such intensity and crystallographic orientation. The specific problem of such correlation was pursued in a study by H. C. Vacher [2], who showed a correlation between extinction positions and the projection azimuth of a cubic axis.

In the present study the relative maximum and minimum intensities of the reflected light were measured photometrically and correlated with crystal orientation. Woodrow, Mott, and Haines [3] used a photometric method but gave no data correlating intensities with orientation. Supplementary experiments were made to provide information concerning the cause of the observed optical anisotropy, which has been the subject of some uncertainty [4].

\section{Theoretical Aspects}

The theory of the reflection of plane polarized light from metallic specimens is one of considerable complexity [5]. This discussion will be limited to a qualitative description of the special case of a plane polarized beam incident normally upon the reflecting surface $[6]$, which is very nearly the case when the metallographic microscope is used with low-powered objectives.

For isotropic metals (cubic structure), normally incident plane polarized light is reflected inherently with neither rotation of the plane of polarization nor introduction of elliptical polarization; therefore,

${ }^{1}$ Italic figures in brackets indicate the literature references at the end of this paper. examination by rotation of the stage of the microscope with crossed nicols exhibits continuous extinction. A like situation exists when the optic axis of the crystal is perpendicular to the surface of a crystal belonging to the uniaxial classification, which includes the tetrogonal, hexagonal, and trigonal systems. If the optic axis and the surface normal do not coincide, it is necessary to consider the orientation of the plane they determine. The intersection of this plane with the surface determines a line called the principal direction, and the incident light vector can be resolved into components parallel to and perpendicular to this direction. Reflectivities and phase changes upon reflection differ for these two components. The reflected light is, in general, elliptically polarized, and the field observed through the crossed analyzer of the microscope is bright. There are, however, four special positions in a complete revolution of the stage where the plane of vibration of the normally incident beam is parallel to or perpendicular to the principal direction in the specimen surface. At these special positions of the stage, the reflected light remains plane polarized in the original plane and is extinguished by the analyzer.

The theory of reflection from orthorhombic, monoclinic, and triclinic materials is of great complexity and will not be discussed here. Fortunately these cases of lower symmetry rarely arise in metallurgical problems.

The above discussion has been confined to the effect on polarized light of the intrinsic optical characteristics of a single flat surface. In view of the possibility, however, that the optical anisotropy of metallographic specimens may be caused by surface contours, the process of multiple reflection of plane polarized light must be considered. To this end, experiments were conducted as will be described later with models made with one, two, or three perpendicular front-surfaced aluminized mirrors.

The polarization figure [7] is another phenomenon that can sometimes be observed with the polarizing microscope and, if distinct, can be used in crystal orientation problems. It resembles the interference 

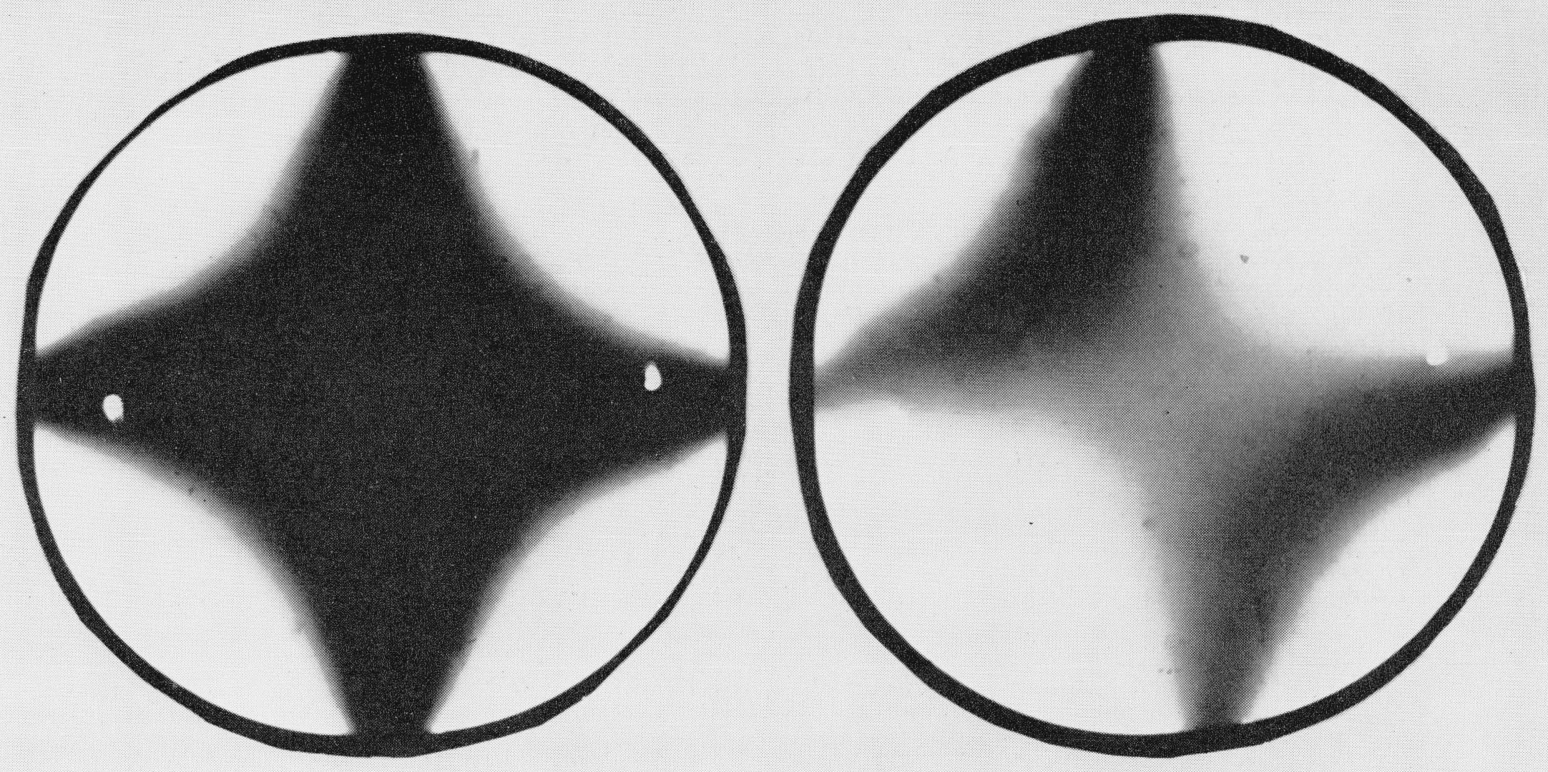

Figure 1. Polarization figures formed by reflection from monel.

Monel contrast solution, $41 \times$ objective, and short focal length telescope as an ocular.

figure [8] observed with convergent plane polarized light passing through transparent anisotropic materials. Instead of arising from path differences caused by birefringence, the polarization figure, according to Cameron and Green [9], results from the rotation and ellipticity introduced in reflected polarized light when the incident beam is not normal to the reflecting surface. The procedure for observing the figures is given in section 3.3. When this procedure is followed, a bright field containing two dark lines, called the isogyres, will be observed. These arise from reflections at those special angles from the surface where, because of particular relations of the planes of the polarizer and analyzer, the angle and plane of incidence of the convergent beam and the optical characteristics of the surface itself, there is a cancelling out of the various factors that disturb the plane polarization in the reflected beam. From those special angles alone, therefore, is the reflected light extinguished by the analyzer. As illustrated in figure 1, the isogyres generally appear as two branches of a hyperbola in the field, but a cruciform figure is formed at four special positions. These are the same positions that give extinctions when the incident light is quasi-normal as is the case with a lowpowered objective. The details, even if only qualitative, of the cause of this phenomenon are rather involved. For a reasonably elementary explanation, the reader is referred to an article by E. N. Cameron and L. H. Green [9], who have studied these figures as they relate to the optical properties of ore minerals.

\section{Materials and Procedures}

\subsection{Materials}

The greatest experimental difficulty encountered in this study was the securing of suitable metallographic specimens with large grains and with surfaces as free as possible from imperfections such as fine scratches, irregular pits or nonuniform films, which would mask the pertinent polarized-light phenomena. In order to avoid errors arising from differences in surface preparation, polycrystalline specimens were used. Many specimens were examined, but the data presented in this report were obtained from four: One each of tin and aluminum and two of monel. The purity of the tin was 99.96 percent and that of the aluminum, 99.99 percent. The monel was commercial grade, which had been heat treated to grow large crystals [2]. The preparation of each specimen is described in section 4 .

\subsection{Determination of Crystal Orientation}

A major preliminary activity was the determination of the crystallographic orientation of each grain used in each specimen. This orientation was defined with respect to the normal to the surface of the specimen and a reference mark on the surface defining zero azimuth.

In the case of monel, these determinations were made by means of angular measurements of the 
traces of twinning planes in two mutually perpendicular surfaces [10]. Monel is a face-centered cubic metal, and the twinning planes are (111) planes. With this information, and with the aid of stereographic plots and standard projections, it was possible to establish the orientations of groups of twinned crystals near the edges of the specimens.

Specimens of aluminum and tin were obtained with grains large enough for the determination of orientations by X-ray diffraction, using the backreflection Laue method with a Greninger net [11, 12]. This method was very simple to employ in the case of the cubic aluminum, but considerable difficulty was encountered with the tetragonal tin. In practice, one pattern alone was not sufficient with the latter to allow the assignment of an unquestioned orientation to a grain. It was necessary to make one or more check patterns with the X-ray-beam incident at an angle to the specimen surface so as to be parallel to some low-index crystallographic direction, according to the tentative interpretation of the first exposure. If the resulting pattern agreed with the proper standard pattern made previously from another grain whose orientation was known with assurance, the orientation of the new grain was considered determined.

\subsection{Measurement of Reflected Polarized Light}

The microscope used in this study uses a Foster prism [13] as the polarizing-analyzing element. The objectives were nominally strain-free. This prism acts as permanently crossed nicols; hence, no effect of slightly uncrossing the analyzer was investigated. The prepared specimen was placed on the rotating stage of this polarizing microscope and set in the zero azimuth position with reference to an edge, grain boundary, or scratch on the surface. A 5.6x objective lens and a 5x ocular were used. By means of stage adjusting screws, a particular grain was centered under the cross lines on the ground glass of the microscope camera. After satisfactory centering and magnification, the bellows was adjusted until the grain under study would cover the photocell aperture. The ground glass was removed and a slide bearing the photomultiplier tube, centered and limited by a $3 / 32$-in.-diameter aperture, was put in its place. The light flux passing into the sensitive tube caused a proportional deflection of the needle on the dial of a commercial electronic photometer. As the specimen was rotated, the intensity of the reflected light would rise and fall in an approximately sinusoidal pattern. The usual procedure was to make three complete revolutions of the stage while recordings were taken of the angular settings of the stage and the photometer current proportional to the light intensity at each of the four maxima and each of the four minima.

It was observed for any one surface that the minimum intensities from all grains were nearly the same, arising from the scattered light from surface imperfections and from the microscope elements. All of the maximum intensities from a grain were averaged together, and the difference taken between that average and the average minimum intensity readings from the grain. This intensity difference, $\Delta I$, is the quantity used in the reported results. Other intensity functions, such as the ratios of various intensities, were examined but were found to be less satisfactory. The angular settings for maximum intensity and minimum intensity (extinctions) were also averaged in a manner to allow for their distribution throughout the complete circle, yielding a grand average value of the first quadrant extinction angle, which was based upon all 24 angular readings. Through the use of results based on averages of large numbers of readings, personal errors were minimized. The extinction angle is defined as the angle from the fiducial line to the position of minimum reflection. If, moreover, polarization figures could be observed (as they readily were with the monel specimens), their crossing positions were also averaged into the value of the extinction angle for each grain. To make these figures visible, the incident light was made highly convergent by the use of a high-powered objective, such as 41x. Moreover, as the figure was formed at the back focal plane of the objective, the method of observation was modified by the removal of the eyepiece. Usually the figure could then be seen directly or through a pinhole eyepiece; viewing was often improved by a lens system, such as a short-focus telescope, which allowed focusing in the proper plane.

\section{Results}

\subsection{Tin}

The tin specimen was prepared by melting the tin in a $3 / 4$-in. quartz ring resitng on a piece of polished graphite. It was then air-cooled. Approximately 15 specimens were made, resulting in only one instance of a specimen (1144, fig. 2) whose surface contained numerous grains large enough (about 1 $\mathrm{mm}$ in smallest dimension) for convenient determination of orientation by X-ray diffraction.

Considerable difficulty was encountered in preparing the surface of this soft metal. It was never ground on papers but polished with only a diamond abrasive, 0 to 2 microns, on microcloth that has been boiled for 2 hours before being used. Then the specimen was etched in acidified ferric chloride reagent [14]. Figures 3, a and b, show the same surface lightly and heavily etched.

On the surface of the specimen there were 29 grains large enough to give usable back-reflection Laue patterns. The crystallographic orientation of the surface normal for each grain is shown in the stereographic triangle in figure 4 . The stereographic angular coordinates of the optic axis (the $c$-axis, or [001] axis), the azimuth referred to a fiducial line in the surface and the colatitude, or zenith distance relative to the surface normal, are given in table 1 . 

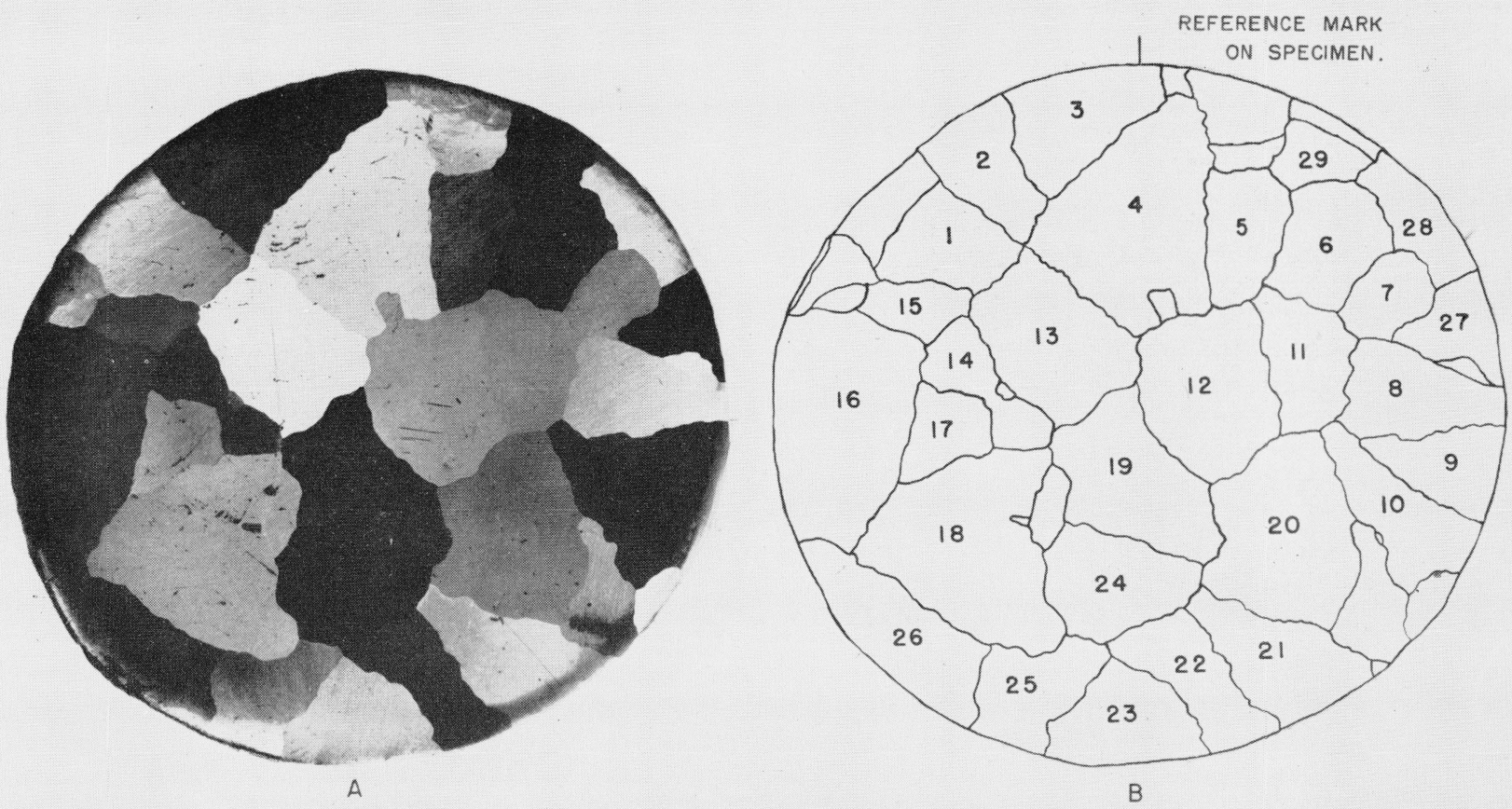

Figure 2. Tin specimen 1144 .

A, Etched with 5-percent ferric chloride solution, approximately $\times 4$; B, sketch for identification of grains.
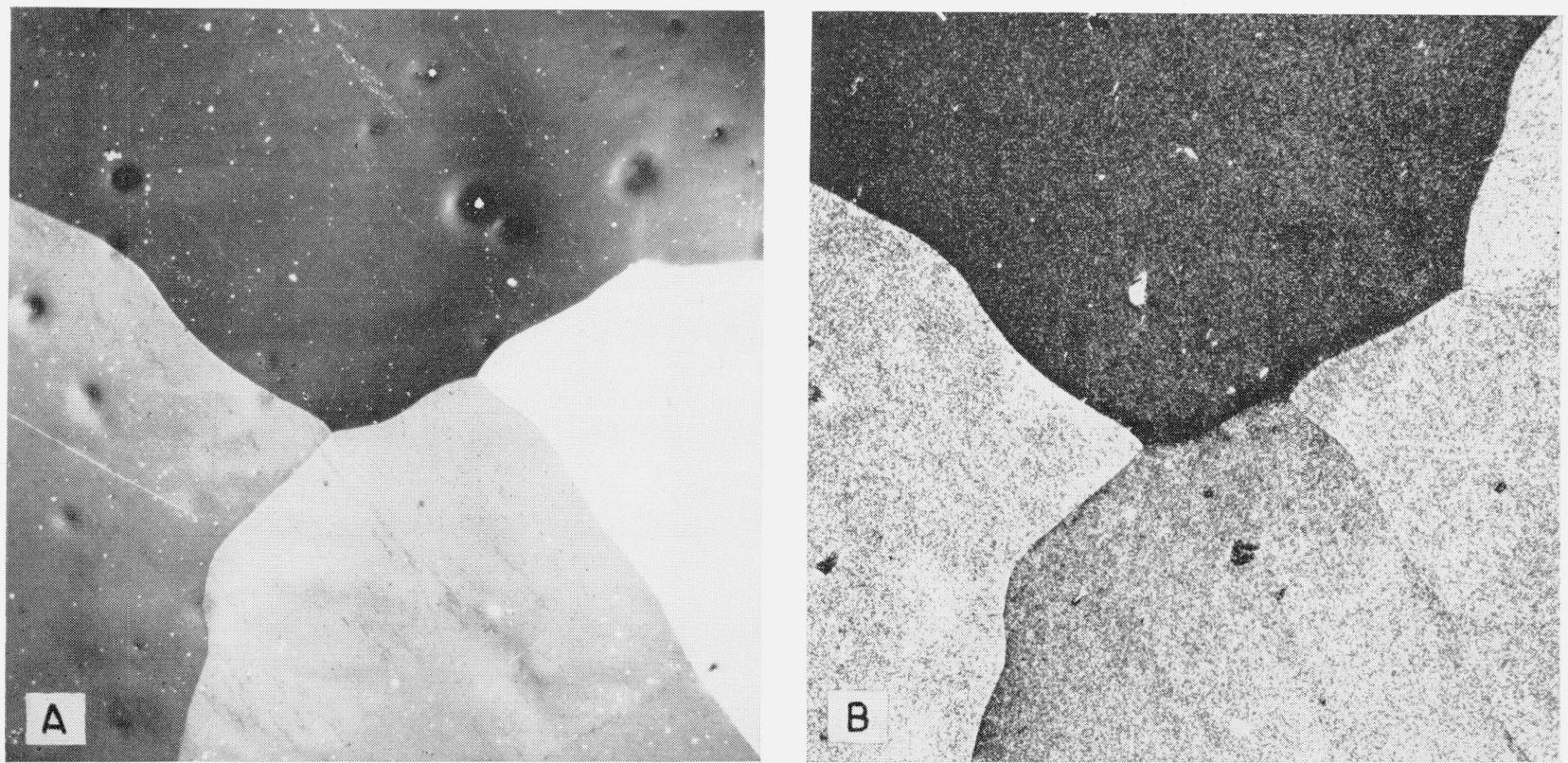

FIGURE 3. Surface of tin 1144 after etching with 5-percent ferric chloride solution.

A, Light etch, $\times 50$; B, deep etch, $\times 50$. 


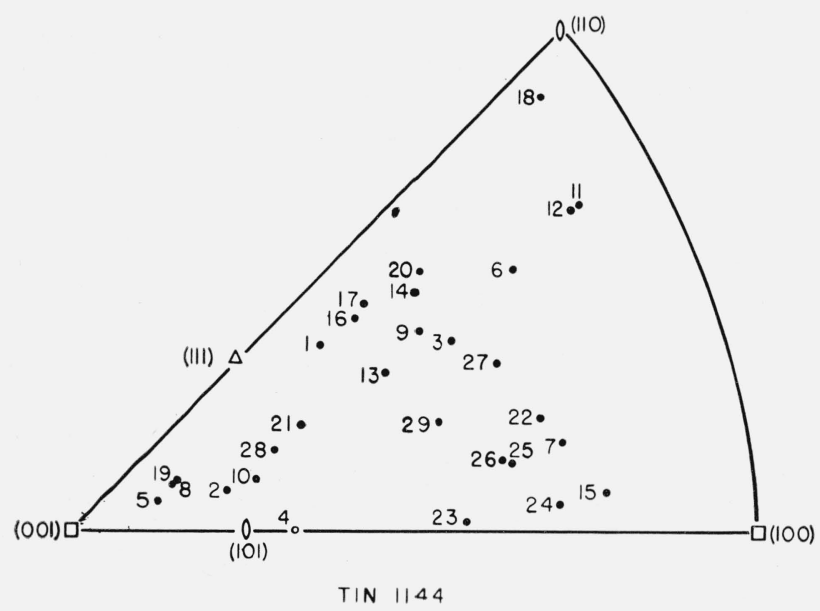

FIGURE 4. Crystallographic orientations of surface normal with respect to individual crystals in the polished surface of tin 1144

Numbers 1 to 29 correspond to the numbers identifying the crystals in figure 2 .

Table 1. Photometer minima, tin 1144, light etch

\begin{tabular}{|c|c|c|c|c|c|c|}
\hline \multirow{2}{*}{ Grain } & \multirow{2}{*}{$\Delta I$} & \multirow{2}{*}{$\psi_{e}$} & \multirow{2}{*}{$\psi_{i}^{\prime}$} & \multicolumn{2}{|c|}{ Orientation } & \multirow{2}{*}{$\Delta \psi$} \\
\hline & & & & $\phi_{c}$ & $\delta$ & \\
\hline $\begin{array}{l}1 \\
2 \\
3 \\
4 \\
5\end{array}$ & $\begin{array}{r}10.4 \\
1.8 \\
25.8 \\
6.9 \\
4.7\end{array}$ & $\begin{array}{c}\text { Degrees } \\
19.6 \\
69.8 \\
69.5 \\
80.7 \\
72.3\end{array}$ & $\begin{array}{c}\text { Degrees } \\
290 \\
250 \\
250 \\
171 \\
72\end{array}$ & $\begin{array}{c}\text { Degrees } \\
289 \\
294 \\
248 \\
186 \\
84\end{array}$ & $\begin{array}{c}\text { Degrees } \\
48 \\
26 \\
63 \\
36 \\
15\end{array}$ & $\begin{array}{r}\text { Degrees } \\
1 \\
-44 \\
2 \\
-15 \\
-12\end{array}$ \\
\hline $\begin{array}{l}6 \\
7 \\
8 \\
9 \\
10\end{array}$ & $\begin{array}{r}33.7 \\
36.6 \\
3.2 \\
26.6 \\
1.9\end{array}$ & $\begin{array}{r}65.4 \\
21.9 \\
80.0 \\
67.2 \\
6.6\end{array}$ & $\begin{array}{l}245 \\
112 \\
170 \\
157 \\
277\end{array}$ & $\begin{array}{l}241 \\
113 \\
184 \\
160 \\
280\end{array}$ & $\begin{array}{l}73 \\
72 \\
18 \\
60 \\
31\end{array}$ & $\begin{array}{r}4 \\
-1 \\
-14 \\
-3 \\
-3\end{array}$ \\
\hline $\begin{array}{l}11 \\
12 \\
13 \\
14 \\
15\end{array}$ & $\begin{array}{l}39.2 \\
39.8 \\
21.4 \\
25.2 \\
43.4\end{array}$ & $\begin{array}{r}51.8 \\
53.3 \\
61.6 \\
7.8 \\
31.4\end{array}$ & $\begin{array}{l}322 \\
323 \\
332 \\
278 \\
301\end{array}$ & $\begin{array}{l}320 \\
320 \\
326 \\
276 \\
299\end{array}$ & $\begin{array}{l}82 \\
81 \\
54 \\
62 \\
76\end{array}$ & $\begin{array}{l}2 \\
3 \\
6 \\
2 \\
2\end{array}$ \\
\hline $\begin{array}{l}16 \\
17 \\
18 \\
19 \\
20\end{array}$ & $\begin{array}{r}14.6 \\
19.9 \\
42.8 \\
1.6 \\
29.8\end{array}$ & $\begin{array}{r}52.3 \\
5.0 \\
82.7 \\
0.8 \\
55.1\end{array}$ & $\begin{array}{r}232 \\
275 \\
263 \\
91 \\
55\end{array}$ & $\begin{array}{r}228 \\
272 \\
260 \\
120 \\
53\end{array}$ & $\begin{array}{l}54 \\
56 \\
85 \\
19 \\
64\end{array}$ & $\begin{array}{r}4 \\
3 \\
3 \\
-29 \\
2\end{array}$ \\
\hline $\begin{array}{l}21 \\
22 \\
23 \\
24 \\
25\end{array}$ & $\begin{array}{l}12.0 \\
47.2 \\
39.4 \\
40.7 \\
33.3\end{array}$ & $\begin{array}{r}1.4 \\
12.5 \\
30.7 \\
87.8 \\
27.8\end{array}$ & $\begin{array}{r}181 \\
12 \\
121 \\
268 \\
208\end{array}$ & $\begin{array}{r}172 \\
12 \\
122 \\
268 \\
213\end{array}$ & $\begin{array}{l}40 \\
70 \\
76 \\
78 \\
71\end{array}$ & $\begin{array}{r}9 \\
0 \\
-1 \\
0 \\
-5\end{array}$ \\
\hline $\begin{array}{l}26 \\
27 \\
28 \\
29 \\
29\end{array}$ & $\begin{array}{r}24.8 \\
25.5 \\
2.1 \\
24.7\end{array}$ & $\begin{array}{r}34.2 \\
68.1 \\
42.2 \\
1.2\end{array}$ & $\begin{array}{r}214 \\
338 \\
312 \\
91\end{array}$ & $\begin{array}{r}216 \\
336 \\
304 \\
92\end{array}$ & $\begin{array}{l}71 \\
67 \\
35 \\
58\end{array}$ & $\begin{array}{r}-2 \\
2 \\
8 \\
-1\end{array}$ \\
\hline
\end{tabular}

Average $|\Delta \psi|=6.3^{\circ}$

If we arbitrarily take $\Delta I=3$ as the value below which valid judgments of extinction angle cannot be made, grains $2,10,19$, and 28 are excluded, and

A verage $|\Delta \psi|=4.0^{\circ}$.

The following symbols are used in the tables:

$\Delta I=$ Change of intensity of light falling on sensitive tube as grain is rotated from a position giving maximum intensity to an extinction position.

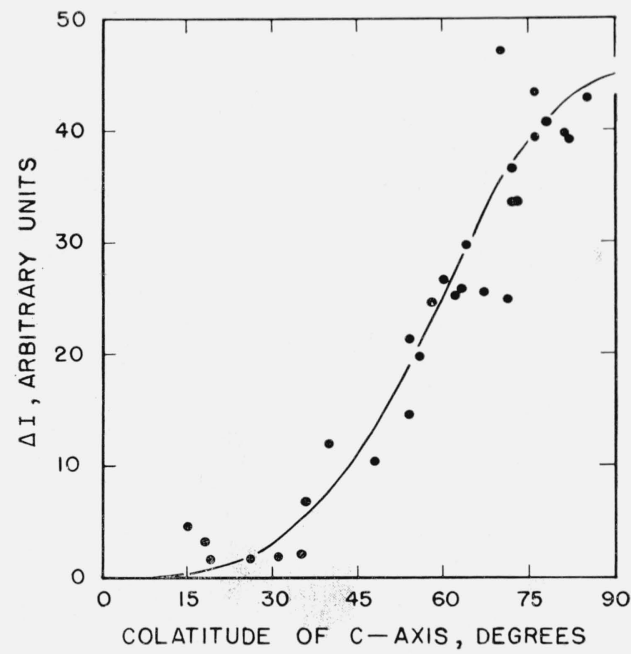

FIgure 5. Relation of $\Delta I$ to angle between surface normal and c-axis (colatitude) in grains of lightly etched tin 1144.

$\psi_{e}=$ Extinction angle: The average angle obtained by a rotation of the microscope stage from a reference line on the surface of the specimen to the position of the first extinction.

$\phi_{c}=$ Azimuth of the $c$-axis (the optic axis) of tin, measured from the same fiducial line as that used in measuring extinction angles.

$\phi_{a}=$ Azimuth of the projection of the cubic axis making the greatest angle with the surface normal.

$\psi_{e}^{\prime}=$ Adjusted extinction angle: $\psi_{e}$ plus such a multiple of $90^{\circ}$ as to give a value near $\phi_{c}$ or $\phi_{a}$, as the case might be.

$\psi_{0}=$ Adjusted average of extinction angle and polarization figure crossings when the latter were usable.

$\Delta \psi=$ Difference between the adjusted extinction angle and the azimuth of the active axis; $\psi_{e}^{\prime}$ or $\psi_{0}$ minus $\phi_{c}$ or $\phi_{a}$, as the case might be.

$\delta=$ Colatitude of the active axis: The angle between the active axis and the normal to the surface.

$\alpha=$ Angle between surface normal and [110] direction in $\mathrm{Al}$.

$\beta=$ Angle between surface normal and [111] direction in $\mathrm{Al}$.

$\gamma=$ Angle between surface normal and [100] direction in $\mathrm{Al}$.

Table 1 also includes the photometer data and the extinction angles for the lightly etched specimen, with the differences between the $c$-axis azimuths and the extinction angles. The average difference was $6.3^{\circ}$. However, if $\Delta I=3$ is arbitrarily taken as the value below which valid judgments of extinction angle cannot be made, four grains are excluded, and the average difference is $4.0^{\circ}$. The results show that, with the exception of these four dim grains, there was excellent agreement of extinction angle with azimuth of the $c$-axis. Figure 5 is a graph of 


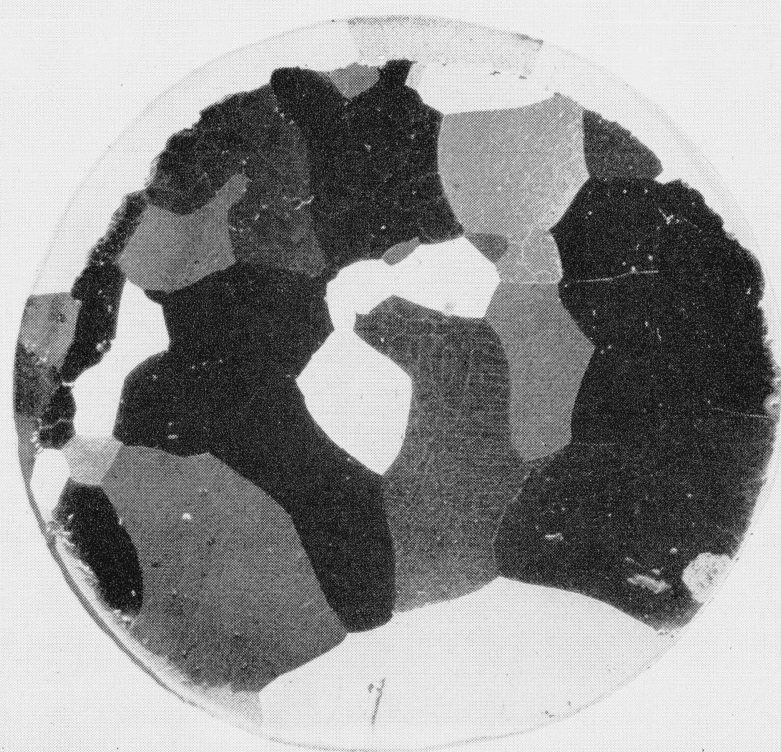

A

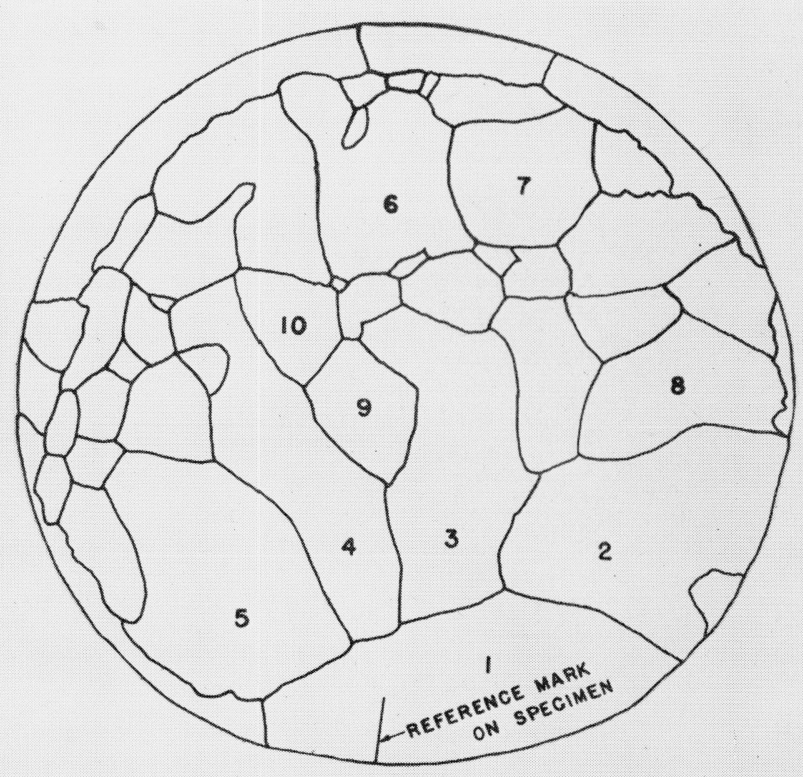

B

Figure 6. Aluminum specimen 1143.

A, Etched with Tucker's reagent, $\times 4$; B, sketch for identification of grains.
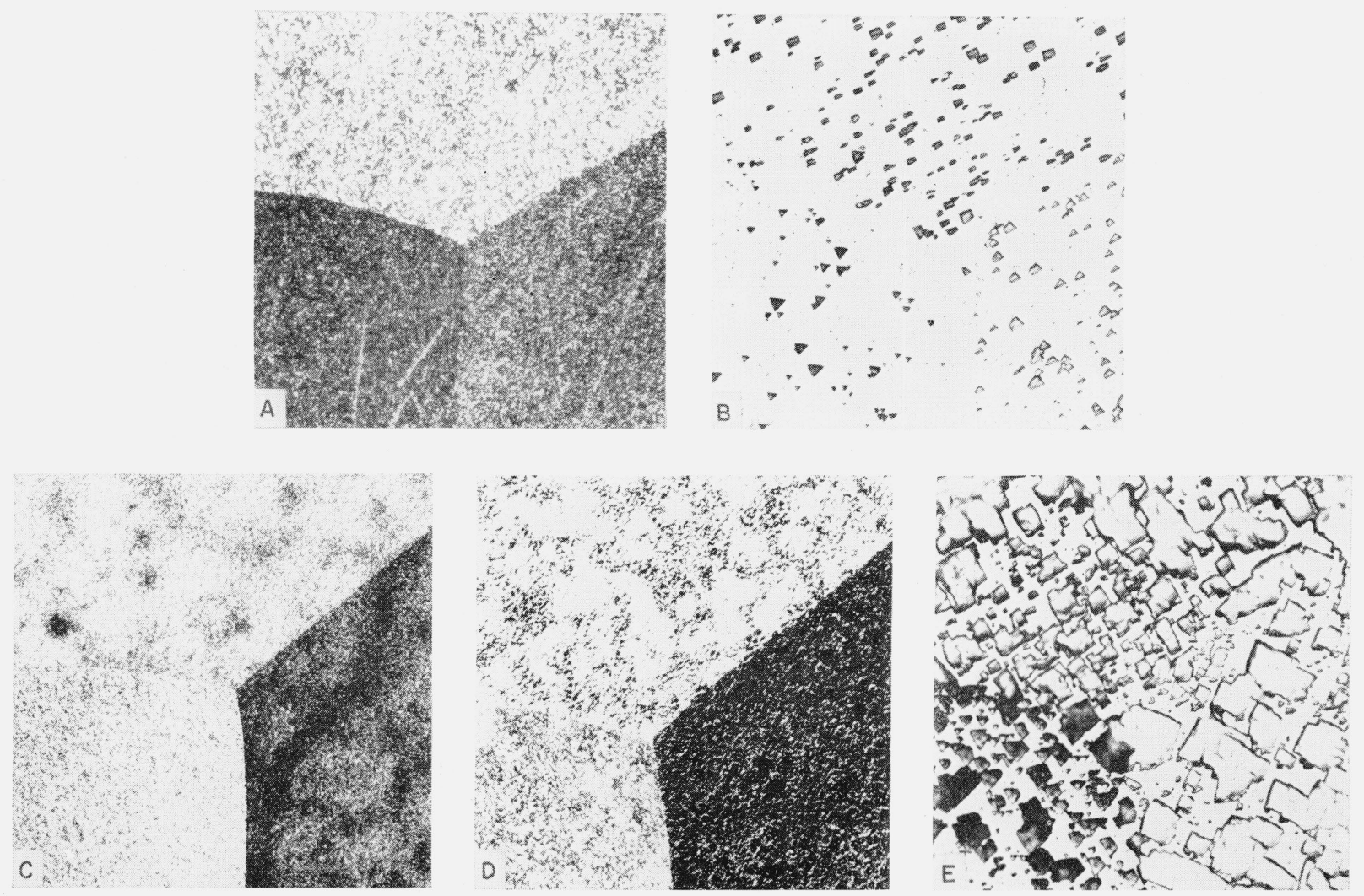

FIGURE 7. Surface of aluminum 1143 after certain treatments.

A and B, Etched with Tucker's reagent, $\times 50$ and $\times 500$, respectively; , anodized, $\times 50 ; \mathrm{D}$ and E, etched with modified Tucker's reagent, $\times 50$ and $\times 500$, 
$\Delta I$ versus $\delta$, the angle between the surface normal and the $c$-axis for this specimen of tin. The curve drawn was for the equation

$$
\Delta I=45 \sin ^{4} \delta
$$

for which no theoretical justification was attempted. However, it exhibited reasonable symmetry and behavior at the end points, and the probable error in the value of the colatitudes of the data points compared with those of points on this curve was less than $5^{\circ}$.

Measurements were also taken from specimen 1144 after the surface had been deeply etched. The average difference between extinction angle and $c$-axis azimuth more than doubled, and the $\Delta I$ relationship to the $c$-axis colatitude developed so much scatter that it could hardly be called more than a trend.

\subsection{Aluminum}

The aluminum specimen, 1143, was a coarsegrained cast specimen that had been polished by conventional metallographic procedure. Its granular structure is shown in figure 6 . The intensity measurements were made after treating the polished surface in four ways: surface 1 was the result of etching with Tucker's reagent [15]; surface 2 was, after repolishing, anodized in an electrolyte recommended by Pearson, Machland, and Hay [16]; surface 3 was a repetition of the anodizing treatment; and surface 4 was the result of a deep etch with a modified Tucker's reagent [17]. These surfaces may be seen in figure 7. To show that the faces of the deep pits in this case were parallel to cubic planes, the positions of the specular reflections of ordinary light from them were measured with an optical goniometer [18]. The results checked satisfactorily.

The crystallographic orientation of the surface normal for the aluminum grains is shown in the stereographic triangle, figure 8 . The stereographic

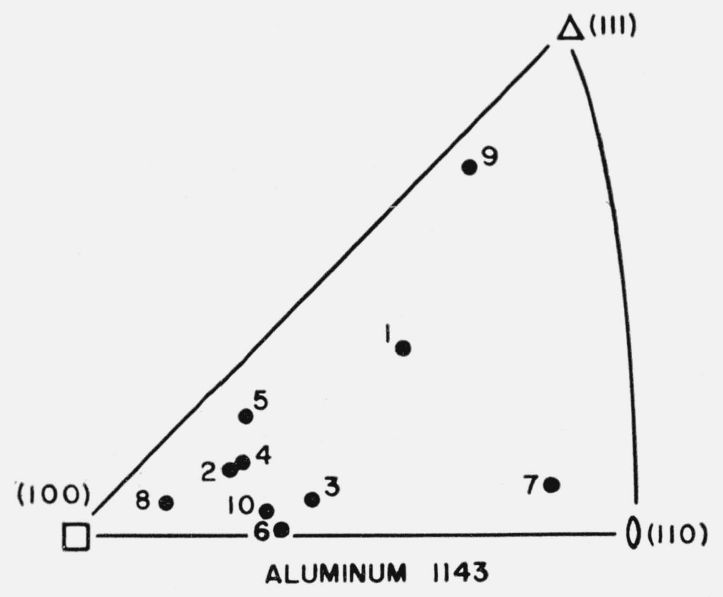

FIgURE 8. Crystallographic orientations of surfaces normal with respect to individual crystals in the polished surface of aluminum 1143.

Numbers 1 to 10 correspond to the numbers identifying the crystals in figure 6 . coordinates, azimuth and colatitude, for the cubic axis making the greatest angle with surface normal, are given with photometer data from surface 4 in table 2 . Table 3 presents the intensity and orientation relationship, which is shown graphically in figure 9.

For the etch-pit conditions, surfaces 1 and 4 , the agreement between the extinction angle and the

TABle 2. Photometer minima Al 1143 , second etch pit condition

\begin{tabular}{|c|c|c|c|c|c|c|}
\hline \multirow{2}{*}{ Grain } & \multirow{2}{*}{$\Delta I$} & \multirow{2}{*}{$\psi_{e}$} & \multirow{2}{*}{$\psi^{\prime}$} & \multicolumn{2}{|c|}{ Orientation } & \multirow{2}{*}{$\Delta \psi$} \\
\hline & & & & $\phi_{a}$ & $\delta$ & \\
\hline $\begin{array}{l}1 \\
2 \\
3 \\
4 \\
5 \\
6 \\
7 \\
8 \\
9 \\
10\end{array}$ & $\begin{array}{c}29.6 \\
65.8 \\
90.5 \\
27.8 \\
45.3 \\
165 \\
201 \\
59.1 \\
22.1 \\
107\end{array}$ & $\begin{array}{c}\text { Degrees } \\
17.2 \\
62.7 \\
84.0 \\
57.5 \\
23.6 \\
59.8 \\
29.9 \\
11.0 \\
80.4 \\
54.9\end{array}$ & $\begin{array}{c}\text { Degrees } \\
17 \\
63 \\
354 \\
148 \\
24 \\
150 \\
120 \\
281 \\
350 \\
325\end{array}$ & $\begin{array}{c}\text { Degrees } \\
19 \\
62 \\
357 \\
147 \\
21 \\
147 \\
119 \\
281 \\
355 \\
325\end{array}$ & $\begin{array}{c}\text { Degrees } \\
75 \\
83 \\
87 \\
84 \\
80 \\
90 \\
86 \\
88 \\
63 \\
88\end{array}$ & $\begin{array}{c}\text { Degrees } \\
-2 \\
+1 \\
-3 \\
+1 \\
+3 \\
+3 \\
+1 \\
0 \\
-5 \\
0\end{array}$ \\
\hline \multicolumn{7}{|c|}{ Average $|\Delta \psi|=1.9^{\circ}$. } \\
\hline
\end{tabular}

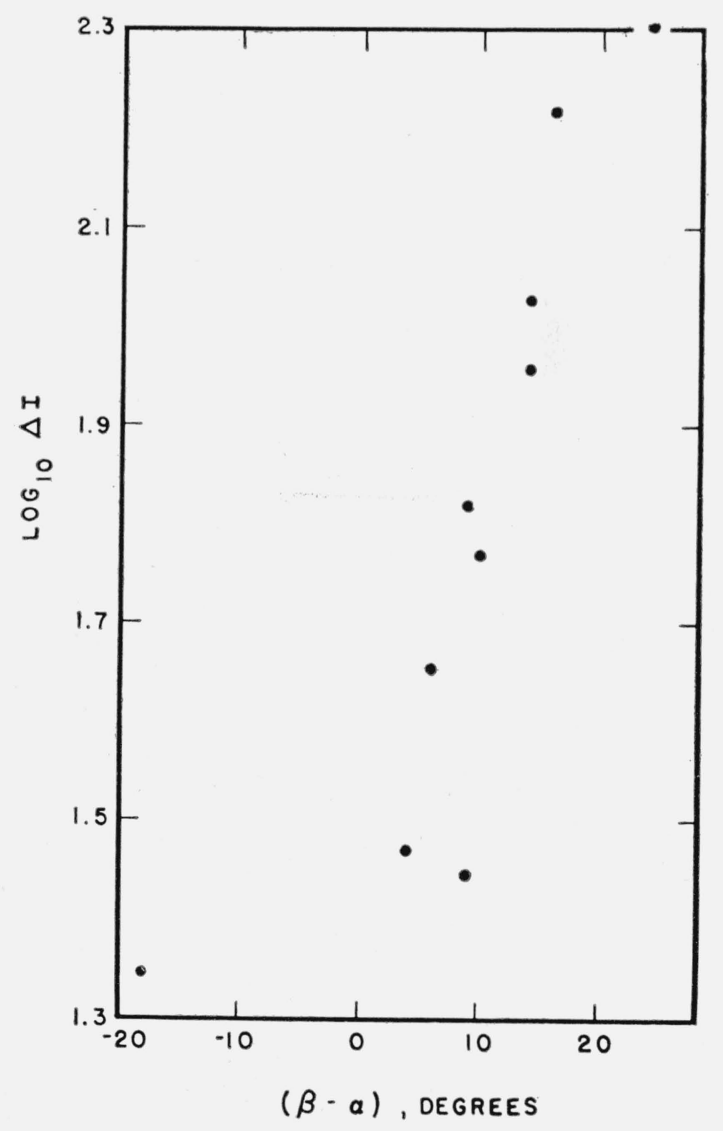

FiguRE 9. Relation of $\Delta I$ of aluminum grains to the difference in angles between surface normal and the nearest [110] and [111] axes. 
TABLE 3. Intensity and angle relationships in $\mathrm{Al} 1143$, second etch-pit surface

\begin{tabular}{|c|c|c|c|c|c|c|}
\hline Grain & $\Delta I$ & $\alpha$ & $\beta$ & $\gamma$ & $\beta-\alpha$ & $\log _{10} \Delta I$ \\
\hline $\begin{array}{l}7 \ldots \ldots \\
6 \ldots \\
10 \ldots \ldots \\
3 \\
2 \\
8 \\
5 \\
1 \\
4 \\
4 \\
9\end{array} \ldots$ & $\begin{array}{r}201 \\
165 \\
107 \\
90.5 \\
65.8 \\
59.1 \\
45.2 \\
29.6 \\
27.8 \\
22.1\end{array}$ & $\begin{array}{c}\text { Degrees } \\
8 \\
28 \\
29 \\
26 \\
33 \\
38 \\
32 \\
22 \\
32 \\
29\end{array}$ & $\begin{array}{c}\text { Degrees } \\
32 \\
44 \\
43 \\
40 \\
42 \\
48 \\
38 \\
26 \\
41 \\
11\end{array}$ & $\begin{array}{c}\text { Degrees } \\
39 \\
17 \\
16 \\
20 \\
14 \\
8 \\
17 \\
31 \\
15 \\
43\end{array}$ & $\begin{array}{c}\text { Degrees } \\
24 \\
16 \\
14 \\
14 \\
9 \\
10 \\
6 \\
4 \\
9 \\
-18\end{array}$ & $\begin{array}{l}2.3032 \\
2.2175 \\
2.0294 \\
1.9566 \\
1.8182 \\
1.7716 \\
1.6561 \\
1.4713 \\
1.4440 \\
1.3440\end{array}$ \\
\hline
\end{tabular}

aizmuth of the cubic axis of greatest colatitude was good. The average difference for surface 1 was $4.2^{\circ}$ and for surface $4,1.9^{\circ}$. No doubt the intensity was related in some way to the orientation of the surface with respect to the grain's crystallography, but no empirical relationship could be found that was satisfactory over the entire range of the limited amount of data at hand. For convenience, one treatment of this data is presented in figure 9 . However, no real functional relationship is implied.

The qualitative aspects of the reflection of plane polarized light from the aluminum with etch pits correlated well with the multiple reflections observed from the pit models made with front-surfaced mirrors because the faces of the etch pits are but slightly imperfect cubic planes.

A few limited experiments were made with three mirror models. Onewas a single-plane front-surfaced aluminized mirror with a stem normal to the plane. One was a "cube corner," three mutually perpendicular mirrors, with a stem making equal angles with the faces. The third consisted of two mirrors at right angles with a stem perpendicular to their line of intersection and making equal angles with each face. From experiments conducted with these models, it appears that there is a useful analogy between the orientation of the plane of the reflected polarized light and the orientation of the image of a linear object multiply reflected by the same mirror system.

Reflection from the single-plane mirror, normal incidence, showed no rotation of the plane of polarization. Nor did reflection from the three mutually perpendicular plane mirrors, no matter what the position of the model as it was rotated about its stem, corresponding to a [111] direction. Study of the light reflected from the model made of two perpendicular mirrors, however, was more complex. When the line of intersection (corresponding to a cubic axis) of the two surfaces was parallel to or at right angles to the plane of polarization of the incident light, the plane was not changed upon reflection. When the line of intersection was at $45^{\circ}$ to the plane of the incident light, the plane of the reflected light was rotated $90^{\circ}$. Or, in general, for a rotation of $\theta^{\circ}$ of the model about its stem, corresponding to a [110] direction, there was a $2 \theta^{\circ}$ rotation of the plane. These results were implied in the equations pertaining to multiple reflection of polarized light published
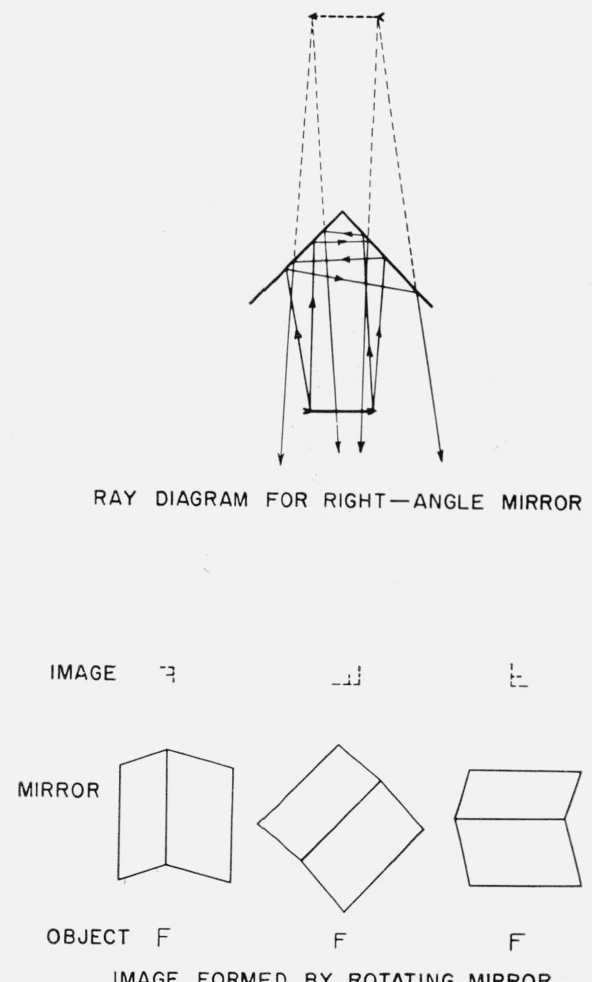

Figure 10. Image formation by right-angle mirror.

by Olwen Jones in 1924 [19]. This turning of the plane of polarization is analogous to the rotation of the image of an object placed before such a rotating mirror, as illustrated in figure 10 .

In observations with the etched specimen, the greatest $\Delta I$ was observed from grains with their surface normals near [110]. In accordance with reflection from the two-mirror model, the intensity of the light passing through the crossed analyzer rose and fell four times per revolution. In the metallographic specimen, the grain with its surface normal nearest [111] had the smallest $\Delta I$, that is, gave the smallest increase in light above the faint light at extinction. This agrees well with the three-mirror model, which did not rotate the plane of polarization in any position, and therefore always showed extinction when viewed through a crossed analyzer. The results of the experiments with the mirror models would lead one further to expect a minimum $\Delta I$ from grains with normals near [100]. This, however, was not observed to be very pronounced. A possible explanation could be that pits of this type were subject, on the average, to a greater degree of imperfection because more cubic faces would be involved in each such pit than in the two simpler cases.

The anodized films, surfaces 2 and 3, were reasonably light, just heavy enough to give smooth, nearly uniform cover. Polarized light, upon reflection from these surfaces was markedly affected, both as 


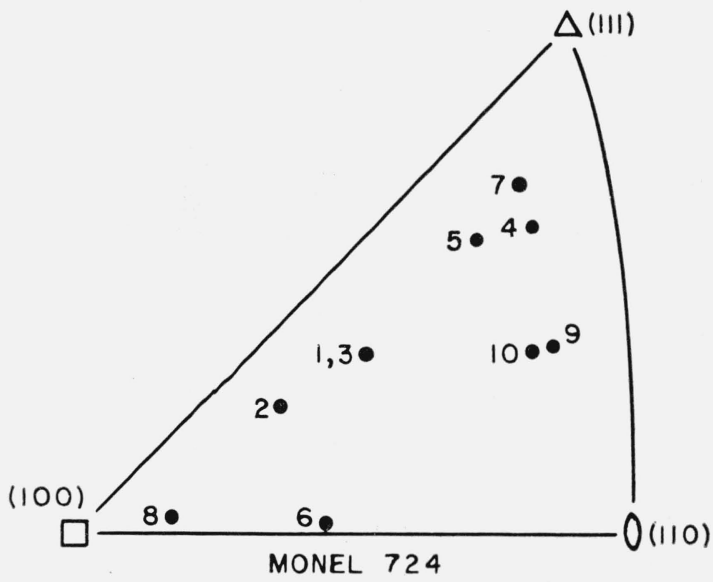

Figure 11. Crystallographic orientations of surface normal with respect to individual crystals in the polished surface of monel 724.

to extinctions and to striking colors when examined through the sensitive tint plate. There was no apparent correlation, however, between this activity and the crystallographic orientation of the underlying metal. A film-orientation correlation was reported, however, by Pearson, Machland, and Hay [16], employing a heavier film than was used in this study.

\subsection{Monel}

The surfaces of the two monel specimens, 724 and 1138 , were polished mechanically and etched with two reagents: A, a cyanide-persulate aqueous solution and B, monel contrast solution [20]. Each solution was used twice alternately on specimen 724 ; only the cyanide solution was used on specimen 1138 . As pointed out in section 3.2, orientations of grains used were determined by the twin-trace method; the positions of the surface normal relative to the crystallographic directions in the grains are shown in stereographic triangles in figures 11 and 12 . The stereographic coordinates, azimuth and colatitude, of the cubic axis of greatest colatitude are given in table 4 for monel 724 , along with photometer data and extinction angles based both on photometer minima and polarization figure crossings. These data were taken from the surface with the second cyanide etch, preparation 3 . Table 5 compares the intensity of reflection and the precision of the cubic axis azimuth-extinction angle correlation for the four surface preparations of this specimen. Table 6 lists the grains in the four cases in order of decreasing $\Delta I$. It is apparent that the several sets of measurements of reflection showed much variation, especially in terms of intensity and its change, $\Delta I$. In all four cases there was reasonable agreement of extinction angle with the azimuth of the cubic axis of greatest colatitude, but the average differences in the cases where the cyanide etch was used were less than half

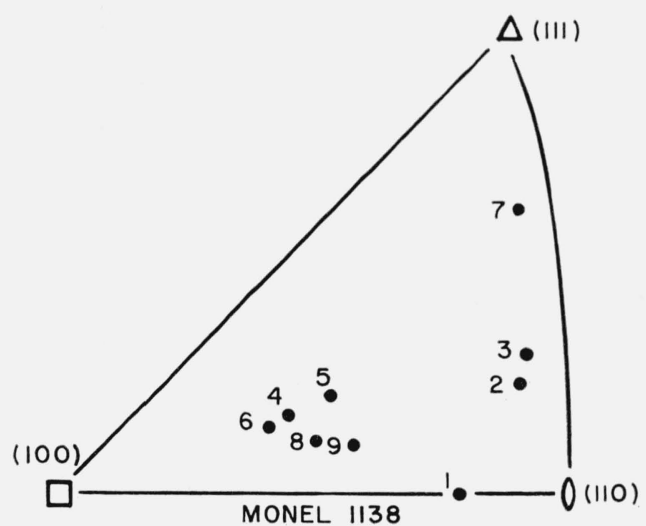

Figure 12. Crystallographic orientations of surface normal with respect to individual crystals in the polished surface of monel 1138 .

TABLE 4. Optical data, monel 724, surface III

\begin{tabular}{|c|c|c|c|c|c|}
\hline \multirow{2}{*}{ Grain } & \multirow{2}{*}{$\Delta I$} & \multirow{2}{*}{$\psi_{0}$} & \multicolumn{2}{|c|}{ Orientation } & \multirow{2}{*}{$\Delta \psi$} \\
\hline & & & $\phi_{a}$ & $\delta$ & \\
\hline $\begin{array}{l}1 \ldots \\
2 \ldots \\
3 \ldots \\
4 \ldots \\
5 \ldots\end{array}$ & $\begin{array}{r}21.6 \\
7.9 \\
29.0 \\
5.8 \\
5.1\end{array}$ & $\begin{array}{c}\text { Degrees } \\
27 \\
347 \\
286 \\
323 \\
357\end{array}$ & $\begin{array}{c}\text { Degrees } \\
34 \\
345 \\
280 \\
321 \\
3\end{array}$ & $\begin{array}{c}\text { Degrees } \\
77 \\
79 \\
76 \\
67 \\
68\end{array}$ & $\begin{array}{c}\text { Degrees } \\
-7 \\
2 \\
6 \\
2 \\
-6\end{array}$ \\
\hline $\begin{array}{l}6 \\
7 \\
8 \\
9 \\
10\end{array}$ & $\begin{array}{r}49.5 \\
5.3 \\
1.8 \\
43.5 \\
23.1\end{array}$ & $\begin{array}{r}250 \\
346 \\
207 \\
82 \\
172\end{array}$ & $\begin{array}{r}251 \\
353 \\
214 \\
83 \\
168\end{array}$ & $\begin{array}{l}89 \\
55 \\
89 \\
76 \\
76\end{array}$ & $\begin{array}{r}-1 \\
-7 \\
-7 \\
-2 \\
4\end{array}$ \\
\hline
\end{tabular}

TABLE 5. Comparison of approximate intensity of reflection and precision of results with various surfaces on monel 724

\begin{tabular}{|c|c|c|c|c|}
\hline Surface & Etch & $\begin{array}{l}\text { Maximum } \\
\quad \text { I for } \\
\text { grain } 9\end{array}$ & $\begin{array}{l}\Delta I \text { for } \\
\text { grain } 9\end{array}$ & $\begin{array}{c}\text { A verage } \\
|\Delta \psi|\end{array}$ \\
\hline $\begin{array}{l}1 \\
2 \\
3 \\
4\end{array}$ & $\begin{array}{l}\text { Cyanide }- \text { - } \\
\text { Contrast. - } \\
\text { Cyanide - } \\
\text { Contrast_- }\end{array}$ & $\begin{array}{r}50.5 \\
21.5 \\
51.2 \\
285.0\end{array}$ & $\begin{array}{r}43.7 \\
20.5 \\
43.5 \\
279.0\end{array}$ & $\begin{array}{c}\text { Degrees } \\
5.1 \\
10.2 \\
4.4 \\
13.0\end{array}$ \\
\hline
\end{tabular}

TABLE 6. Grains of monel 724 ranked by intensity values.

\begin{tabular}{|c|c|c|c|c|}
\hline \multicolumn{5}{|c|}{ Surface condition } \\
\hline Rank & $\begin{array}{c}1 \\
\text { grain }\end{array}$ & $\stackrel{2}{2}$ grain & $\stackrel{3}{\text { grain }}$ & $\stackrel{4}{\text { grain }}$ \\
\hline 1. & 9 & 6 & 6 & 9 \\
\hline 2. & 1 & 9 & 9 & 10 \\
\hline 3 & 6 & 10 & 3 & 1 \\
\hline 4 & 3 & 3 & 10 & 7 \\
\hline $5 \ldots$ & 10 & 5 & 1 & 3 \\
\hline $6 \ldots$ & 5 & 1 & 2 & 4 \\
\hline 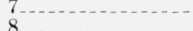 & $\begin{array}{l}7 \\
2\end{array}$ & $\frac{7}{2}$ & $\frac{4}{7}$ & $\begin{array}{l}5 \\
2\end{array}$ \\
\hline 9 & 4 & 4 & 5 & 6 \\
\hline $10 \ldots$ & 8 & 8 & 8 & 8 \\
\hline
\end{tabular}


TABLE 7. Photometer minima for grains of Monel 1138

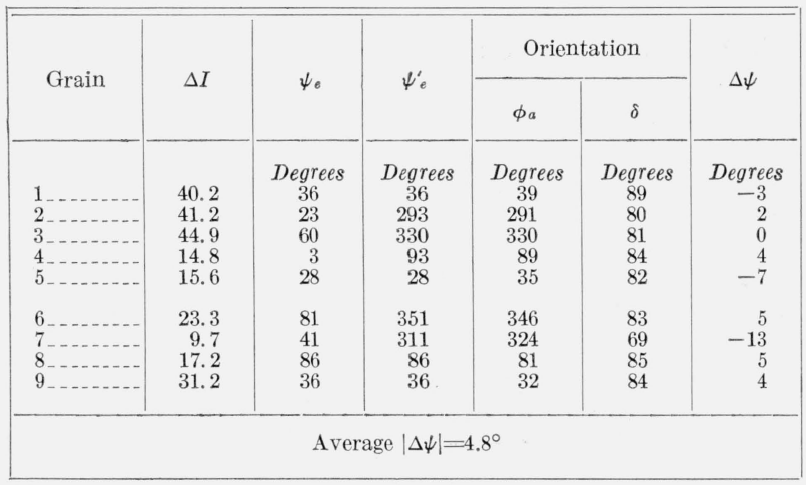

as large as those in the contrast etch cases. In all cases, however, there were large shifts in ranking the grains in terms of intensity, as can be seen in table 7 . Hence, there was obviously no correlation of intensity with orientation, except the vague observation that the grain with surface normal nearest the [100] direction was always of smallest $\Delta I$, whereas the two grains with normals nearest [110] were always near the top of the list in $\Delta I$.

The other monel specimen, 1138, was etched with the cyanide reagent only. The surface normals of the grains are located in the stereographic triangle in figure 12, and the coordinates of the cubic axis of greatest colatitude are given in table 7 , along with the photometer and microscope data. These results are comparable with those from monel 724 , showing good agreement of extinction position with the azimuth of the cubic axis of greatest colatitude, and only a hint of correlation of intensity with angles between the surface normal and the [110] direction.

It should be stated that the correlations were usually poor when the normals to the surface were far from the [110] direction. This was observed for several grains in a different area of specimen 1138, for which data are not presented here. The extinction angles had a fair degree of reproducibility but did not always correspond with the azimuths of the cubic axis of greatest colatitude. In these cases they often corresponded to the azimuths of the axis most nearly normal to the surface. This was in agreement with some of the observations reported in the paper by Vacher [2], where specific data on a few such grains with anomalous extinctions were presented. This disturbing behavior did not appear in the case of aluminum or tin.

\section{Discussion}

The practical value of the polarizing microscope for the determination of the orientation of crystal grains in metallographic specimens and the source or mechanism of the optical anisotropy will be discussed in the light of the foregoing results.

\subsection{Value of Polarized-Light Measurements in Determining Crystal Orientation}

An examination of the results presented above reveals varying degrees of correlation between certain characteristics of reflected polarized light and the crystallography of the grains in the reflecting metal surface. It is true, however, that a complete orientation determination of a grain in a metallographic specimen cannot be made alone from polarizedlight observations such as these. The first difficulty encountered is the obtaining of a suitable specimen surface, that will not cause the pertinent phenomena to be masked by the extraneous optical effects of scratches, irregular pits, nonuniform films, coldworking, inadequate etching, etc. If a nearly perfect, lightly etched surface with polarizing activity is obtained, the observation with crossed polarizing elements of the four extinction angles as the specimen is rotated will give within a few degrees four possible positions $90^{\circ}$ apart for the azimuth of the "active" axis. This is the optic axis of a uniaxial material, or the axis making the greatest angle with the normal for a material showing anisotropic effects due to etch pits with facets parallel to cubic planes; or stated another way, when the polarizingly active specimen is in an extinction position on the microscope stage, the plane containing the surface normal and the active axis will coincide with either the plane of polarization of the polarizer or that of the analyzer.

If the surface quality is such that polarization figures can be observed, this ambiguity is cut in half. The four settings of the specimen, observed conoscopically, which give the cross figure, correspond to the extinction positions. As the specimen is rotated the isogyres spread apart and move toward the edges of the field in the quadrants containing the projection of the optic axis of a uniaxial material [9]. In this study the observation of polarization figures with uniaxial tin was found to be very difficult, but such observations were made quite successfully with etched monel, where the position of the cubic axis of greatest colatitude governed the behavior of the isogyres just as the optic axis is expected to do in theory. Locating the projection of the active axis in this way reduces its possible azimuth to two positions $180^{\circ}$ apart. Polarization figures were not observed with aluminum either etched or anodized. In general, the degree of surface perfection required for the formation of these figures is even higher than that required for consistent extinction angle observations. Apparently the presence of textured films, pits, etc., precluded their use with the aluminum and tin specimens. The other quantity measured, change of intensity of polarized light as the specimen is rotated, is less reliable in its relation to orientation, probably because of its very great dependence upon surface condition. Nevertheless, if we can generalize from the observations of tin, it appears that for uniaxial materials with carefully prepared surfaces, 
the order of grains ranked in terms of decreasing $\Delta I$ will correspond very closely to the order of those ranked in terms of decreasing colatitude of the optic axis, approximating a fourth-power sine law.

On the other hand, the case for cubic materials is more complex and apparently is subject to more disturbing factors. This is shown by the great variations and inconsistencies of the intensities observed with monel. The observations with etch pits on aluminum were somewhat encouraging, and lead one to suppose that if suitable etchants that consistently create pits with known faces, preferably cubic, are employed [18], empirical relationships might be found for some isotropic materials.

It is apparent that the measurement of extinction angle and intensity is not sufficient to permit one to obtain a complete orientation determination. This procedure can be of great value, however, as an aid when other methods are being employed, as, for example, the twin-trace method or the etch-pit reflection method. Ambiguities often arise in twintrace studies; and, with the method of reflections of ordinary light from etch pits, large deficiencies of precision are often an impediment. The additional information supplied by polarized-light examination will be an aid in resolving the difficulties encountered in such cases. In connection with the back-reflection Laue method, polarized-light data will facilitate the assignment of indices or the positioning of the specimen so that more readily interpreted patterns can be obtained.

\subsection{Cause of the Optical Anistropy of Metals Having Cubic Structure}

In section 1 brief reference is made to the question of the source of the polarization phenomena. Observations reported here showed distinct cases of polarization activity due both to a surface film (the anodized surface) and to surface contour (the etch pits). It is not surprising that an effect on the reflected polarized light can be due to either mechanism. The question would still remain, however, whether the effect of the film on the light was due to intrinsic anisotropy or to underlying metal-surface contour. It has been shown here that, when surface contour (etch pits) alone was involved, there was good correlation of the optical effects and the crystal orientation. The data from lightly anodized surfaces, however, gave no such correlation. It is therefore evident that the anisotropy of these light films was not related in any simple manner to the underlying surface contour, if such contour was a function of the crystal orientation, as in the case of the etch pits. Hence, it seems probable that these films are inherently anisotropic but that their orientation is related in an as yet undefined manner to the orientation of the underlying grains.

The results from monel were very much less definite than those from aluminum, but they showed a slight resemblance to the latter. Their lack of consistency can perhaps be attributed to the variation of degree of attack on cubic faces by the etching reagents.

When working with tin the conditions were somewhat different. The fact that the metal itself was anisotropic was the controlling factor in its behavior. Because of the softness of the metal, a very light etch improved the results by removing disturbing surface features such as polishing scratches, but the heavy etch worked against a correlation because of excessive irregular attack or an accumulation of an obscuring film of etch products.

\section{Summary}

Metallographic specimens of tin, aluminum, and monel were prepared, and the crystallographic orientations of many of the grains in each specimen were determined. The reflection of plane polarized light normally incident upon the specimens after various surface treatments was examined by means of a polarizing microscope and an electronic photometer. In some cases convergent light was used and polarization figures were observed. Very significant correlation was found in most cases between the extinction angle and the azimuth of the active axis. Moreover, in the case of tin, the change of intensity of the light from a grain was approximately proportional to the fourth power of the sine of the angle between the optic axis and the surface normal. In the case of aluminum and monel, the relationship of intensity to orientation was not so clear. The results support the hypothesis that the source of the optical anisotropy of polarized-light reflection from cubic metals is an oriented-surface contour.

The authors acknowledge capable assistance of Ellen A. Buzzard in preparing the metallographic specimens and express their gratitude to Mrs. Buzzard and Ruth E. Dowden for aid in the preparation of many of the drawings and photographs used.

\section{References}

[1] D. H. Woodard, Stages in the deformation of Monel metal as shown by polarized light, Am. Inst. Min. Met. Engrs, Metal Trans, 185, 722 (1949).

[2] H. C. Vacher, A correlation of polarized light extinctions with crystal orientation in 70 nickel-30 copper alloy, J. Research NBS 49, 149 (1952) RP2351.

[3] J. Woodrow, B. W. Mott, and H. R. Haines, Analysis of polarized light reflected from absorbing materials at normal incidence, Proc. Phys. Soc. London [B] 65, 603 (1952).

[4] G. K. T. Conn and F. J. Bradshaw, Polarized light in metallography, p. 72 (Academic Press, Inc., New York, N. Y., 1952).

[5] F. A. Jenkins and H. E. White, Fundamentals of optics, p. 560 (McGraw-Hill Book Co., New York, N. Y., 1950).

[6] B. W. Mott and H. R. Haines, Examination of metals under polarized light. I. Theory and apparatus, Research 4, 24 (1951).

[\%] G. K. T. Conn and F. J. Bradshaw, Polarized light in metallography, p. 66 (Academic Press, Inc., New York, N. Y., 1952). 
[8] F. A. Jenkins and H. E. White, Fundamentals of optics, p. 537 (McGraw-Hill Book Co., New York, N. Y., 1950).

[9] E. N. Cameron and L. H. Green, Polarization figures and rotation properties in reflected light and their application to the identification of ore minerals, Econ. Geol. 45, 719 (1950).

[10] C. S. Barrett, The structure of metals, p. 39 (McGrawHill Book Co., New York, N. Y., 1943).

[11] A. B. Greninger, Determination of orientation of metallic crystals by means of back-reflection Laue photographs, Trans. Am. Inst. Mining Met. Engrs. 117, 61 (1935); A back-reflection Laue method for determining crystal orientation, Z. Krist 91, 424 (1935).

[12] C. S. Barrett, The structure of metals, p. 167 (McGrawHill Book Co., New York, N. Y., 1943).

[13] L. V. Foster, A polarizing vertical illuminator, J. Opt. Soc. Am. 28, 124 (1938).

[14] C. H. Hack and F. H. Clark, The metallography of tin and tin alloys, Metals Handbook, 1948 ed., p. 1069 (Am. Soc. Metals, Cleveland 3, Ohio).
[15] F. Keller, The metallography of aluminum alloys, Metals Handbook, 1948 ed., p. 798 (Am. Soc. Metals, Cleveland 3, Ohio).

[16] E. C. Pearson, G. Machland, and R. H. Hay, An electrolytic etch technique for aluminum and its application to some metallographic problems, Can. Inst. Metals 45, 598 (1952).

[17] G. E. G. Tucker and P. C. Murphy, A method for determining orientation in aluminum single crystals and polycrystalline aggregates, J. Inst. Metals 81, 235 (1953).

[18] C. S. Barrett, The structure of metals, p. 173 (McGrawHill Book Co., New York, N. Y., 1943)

[19] O. Jones, Reflection of plane polarized light by etched metals, Phil. Mag. 48, 207 (1924).

[20] D. I. Sinizer, The metallography of nickel and nickel alloys, Metals Handbook, 1948 ed., p. 1045 (Am. Soc. Metals, Cleveland, Ohio).

Washington, November 3, 1953. 